\title{
Heart failure 2019: insights from the National Society of Cardiology Journals
}

\author{
Plamen Gatzov', Jean-Jacques Monsuez², Gergely Agoston³, Michael Aschermann", \\ Hala Mahfouz Badran ${ }^{5}$, Ariel Cohen ${ }^{6}$, Kurt Huber?, Evgeny Shlyakhto ${ }^{8}$, Dilek Ural', \\ Fernando Alfonso ${ }^{10}$
}

\author{
${ }^{1}$ Editor-in-Chief of Bulgarian Cardiology Journal, Bulgaria \\ ${ }^{2}$ Editor-in-Chief of Archives des maladies du coeur et des vaisseaux pratique, France \\ ${ }^{3}$ Associate Editor of Cardiologia Hungarica, Hungary \\ ${ }^{4}$ Editor-in-Chief of Cor et Vasa Czech Republic, \\ ${ }^{5}$ Editor-in-Chief of Egyptian Heart Journal, Egypt \\ ${ }^{6}$ Editor-in-Chief of Archives of Cardiovascular Diseases, France \\ ${ }^{7}$ Editor-in-Chief of Austrian J Kardiology, Austria \\ ${ }^{8}$ Editor-in-Chief of Russian Journal of Cardiology, Russia \\ ${ }^{9}$ Archives of The Turkish Society of Cardiology, Turkey \\ ${ }^{10}$ Chairman of The Editors Network of the European Society of Cardiology, Spain
}

Address for correspondence:

Prof. Plamen Gatzov, MD, PhD, DSc, FESC, Medical University of Pleven, 1, St. KI. Ohridski Street, 5800

Pleven, Bulgaria.

Email: plamengatzov@yahoo.com

Heart failure (HF) became one of the biggest problems of the health care systems in the developed countries. It's prevalence has the characteristics of pandemic in the European Society of Cardiology (ESC) member countries. The population aging and poorly controlled cardiovascular risk factors such as hypertension, overweight, and diabetes are the most important factors for that situation. The differences in the disease epidemiology, diagnosis and therapy among ESC member countries have been recently well described in the Atlas registry. To understand the specific features in the ESC countries, the ESC Editors Network created the initiative to present the most important publications from the National Societies of Cardiology journals (NSCJ) every year. For the 2019 the decision was the articles to be in the field of HF. The following review presents the selection of such papers.

Keywords: heart failure, European society of cardiology, national societies of cardiology journals, publications

\section{Abbreviations}

adjHR: Adjusted hazard ratio; CAVD: Calcific aortic valve disease; CCU: Coronary care unit; ESC: European cardiology society; GLS: Global longitudinal strain; HF: Heart failure; HFpEF: Heart failure with preserved ejection fraction; HFrEF: Heart failure with reduced ejection fraction; KCCQ: Kansas City Cardiomyopathy Questionnaire; LP(a): Lipoprotein (a); LVEF: Left ventricular ejection fraction; MRI: Magnetic resonance imaging; NSC: National societies of cardiology; NSCJ: National societies of cardiology journals; NYHA: New York Heart Association; SGLT2: Sodium-glucose cotransporter-2; SSc: Systemic sclerosis, TAVI: Transcatheter aortic valve implantation; LS: Liver stiffness 


\section{Introduction}

The prevalence of heart failure (HF) is increasing worldwide as a result of continuous ageing of the population, and unresolved problem of poorly controlled cardiovascular risk factors such as hypertension, overweight, and diabetes (1). Facing these challenges, continuous progress of chronic HF treatment over the recent decades have been achieved concomitantly (2). Several disparities have been observed, however, and the increase in prevalence of those risk factors, and HF respectively depends on many factors such as gender, educational and socioeconomic levels in different geographical areas (3). Differences in prevalence of HF across Europe have been described recently by the Atlas Writing Group to the ESC (4). Adequate HF treatment improves quality of life and survival however optimal guideline-directed therapy of HF including angiotensin-converting enzyme inhibitors, angiotensin-receptors blockers, beta-blockers, and mineralocorticoid receptor antagonists is not applied for many patients, because of similar limiting factors $(1,5)$. The improvement in HF treatment needs the specificities in different ESC member countries to be taken into account. This approach is expected to be best achieved and disseminated to cardiologists by the NSC journals. During the ESC Congress 2019 in Paris, the ESC Editors Network started an initiative to boost the dissemination of cardiology science, published in the NSC journals. The ESC Editors Network members decided the first topic of such review to be the publications in the field of HF.

In 2019 evolving concepts have been highlighted, regarding optimized administration of sacubitril-valsartan with initiation during the index admission for $\operatorname{HF}(6,7)$. The potential benefit of SGLT2 inhibitors in reducing cardiovascular mortality and heart failure among diabetics have been widely reported by international journals $(8,9)$. By contrast, NSC journals reported more specific aspects of HF, mainly devoted to regional epidemiologic aspects, pathogenesis, unusual features for diagnosis, and specific causes of HF and their treatment.

A panel of contributions on HF published by these NSCJ in 2019 has been selected by their respective Editors-in-Chief and commented in this short summary.

\section{Epidemiology}

Epidemiology of HF among ESC member countries has been presented recently by Timmis $A$ et al. (3). The conclusion of the Atlas scientific group is that there are inequalities in the prevalence of risk factors, cardiovascular disease burden, cardiovascular mortality, and some therapeutic methods implementation (coronary interventions, device implantations and cardiac surgery procedures) among the ESC member countries.
These parameters depend on socio-economic factors and mostly are worse in middle-income, compared to high-income countries. More country specific features can be understood by publications from National Societies of Cardiology (NSC). Badran HM et al. in a single center observational study on 1006 patients admitted to the coronary care unit (CCU) estimated the prevalence of HF by gender, preserved or reduced left ventricular ejection fraction (LVEF). They reported higher prevalence of $\mathrm{HF}$ at all and higher incidence of $\mathrm{HF}$ with reduced ejection fraction (HFrEF) in female patients. Female patients were older, more obese, with more co-morbidity but had less acute coronary syndromes and required less percutaneous coronary interventions. Despite those differences the prognosis was similar in female and male patients (10) (Egypt Heart J 2019; 71: 167. Editor-in-Chief: Hala Mahfouz Badran).

\section{Etiology and predisposition}

Calcific aortic valve disease (CAVD) is a disorder of high social significance not only because it is widespread but also because it can progress while clinically unrecognized over a long period of time. After development of severe aortic stenosis, the 2-year survival rate in the absence of surgical intervention is about $50 \%$. Nowadays, the causes of this pathological condition and its exacerbation mechanisms remain unknown. Tomova $V$ et al. tested the hypothesis that the polymorphism rs10455872 at the lipoprotein (a) [LP(a)] gene locus, encoding LP(a) increases the risk of aortic valve disease. One hundred forty six individuals: 108 patients with CAVD and 38 controls were studied. The authors reported that the patients with at least one mutant allele of the gene have four times greater risk for CAVD development. They argued that confirmation of genetic nature of the disease can help to prevent or optimize treatment of this frequently seen disease (11) (The Bulgarian Cardiology Journal - Editor in chief: $P$ Gatzov). In addition modalities for the diagnostic evaluation of transthyretin-amyloidosis have improved significantly over the recent years, using structural screening by magnetic resonance imaging (MRI) assessment, as reported by Kauffmann, et al. (12). The coexistence of both diseases (namely CAVD and transthyretin-amyloidosis) appears to hold important diagnostic and prognostic implications. The value of electrocardiogram, echocardiography, MRI, Technetium scintigraphy and endomyocardial biopsy have been pointed out in this regard. (Austrian J Kardiology - Editor in Chief: $\mathrm{K} \mathrm{Hu}$ ber). On the other hand, subclinical myocardial involvement is common in systemic sclerosis (SSc) and is associated with HF and poor prognosis. In a study of 73 SSc patients, Vertes $V$ et al. tested the 2D-speckletracking-derived global longitudinal strain (GLS) method for early myocardial involvement in the disease. As 
a control group served 23 gender- and age-matched healthy volunteers. Significantly lower GLS values were found in patients compared to volunteers $(-17.2 \pm 2.3 \mathrm{vs.}$ $-18.7 \pm 1.4 \%, p=0.001)$. The GLS correlated also with the duration of the disease from the onset of the Raynaud phenomenon ( $r=0.274 ; p=0.021)$, from the first nonRaynaud symptoms $(r=0.245 ; p=0.039)$ and with the New York Heart Association (NYHA) functional class of the patients $(r=0.242 ; p=0.042)$ (13) (Cardiologia Hungarica Editor in Chief: Gergely Agoston).

\section{Pathogenesis}

In a prospective study including 297 patients Lelyavina TA et al. reported the potential for muscle differentiation, regeneration and growth of satellite skeletal muscle precursor cells obtained from patients with HF with reduced ejection fraction (HFrEF). They concluded that the studied parameters do not differ from those found in healthy donors. This may explain one of the mechanisms by which training walking patients for more than 1,5 hours daily contributes to the development of physiological reverse myocardial remodeling to a greater extent than aerobic training (14) (The Russian Journal of Cardiology, Editor in chief: E Shlyakhto).

\section{Diagnosis of HF}

A number of articles in the NSCJ have been dedicated to diagnostic issues in patients with HF. Vdovenko DV, Libis RA. compared 80 patients (NYHA class I-lla and stage $A-C$ of the $A B C D$ classification of the American College of Cardiology) with chronic HFpEF with 30 healthy controls by using the 6-minutes' walk test and echocardiography. They found that all patients have diastolic dysfunction (60 - abnormal relaxation pattern, and 20 - pseudonormal pattern), reduced global and segmental strain of the left ventricle (Russ $\mathrm{J}$ Cardiol - Editor in chief: E. Shlyakhto). The impact of HF on the other organs has been addressed in the paper of Içen $Y K$ et al. They estimated the liver stiffness (LS) in HF patients. They found that in patients with HFrEF the LS estimated by ElastPQ technique increases when patients are in higher functional class by NYHA. A higher LS was associated with higher right ventricular myocardial performance index, regurgitation pressure gradient, NT-proBNP and aspartate aminotransferase levels (16) (Archives of The Turkish Society of Cardiology - Editor-in-chief: Dilek Ural). Ischemic cardiomyopathy challenges therapy of $\mathrm{HF}$ in many aspects. Assuming that the SYNTAX score is not just a measure of the severity of coronary artery disease, but also of its complexity, Öztürk $S$ in a single center study tested the degree of coronary atherosclerosis, estimated by SYNTAX score and myocardial viability in patients with is- chemic cardiomyopathy. Not surprisingly, patients with a non-viable myocardium had a significantly higher SS compared to those with a viable myocardium (17.6 \pm 3.7 vs. $14.1 \pm 5.2$, respectively; $p=0.004$ ) (17) (Turk Kardiyol Dern Ars, Editor-in-Chief).

\section{Treatment}

Treatment of HF has generated major scientific interest. Clinical implications of HF associated with valvular heart disease has been reported by several NSCJ. Transcatheter aortic valve implantation (TAVI) is a recommended alternative to surgical aortic replacement for the treatment of symptomatic severe aortic stenosis. Indications are now rapidly expanding towards patients at lower surgical risk. Generalization of the transfemoral vascular approach, technological advances and increased operator skills have resulted in higher rates of procedural success and improved short-term and long-term survival. Notwithstanding, patients undergoing TAVI remain burdened with frequent co-morbidities, and readmission within 30 days from the index hospitalization has been reported as a common complication. Symptomatic HF is an important trigger that leads to TAVI. However, data on the incidence of readmission for heart failure after successful TAVI are scarce. A French study on 1139 patients, published by Guedeney, et al. in Archives of Cardiovascular Diseases (Editor in Chief: A. Cohen) reported that readmission for HF occurs in one of 10 patients after successful TAVI, and constitutes a strong risk factor for mortality. In this setting, co-morbidities and LVEF $\leq 35 \%$ after TAVI are the main risk factors for readmission for HF. Adjusted hazard ratio (adjHR) of LVEF $\leq 35 \%$ was $2.12,95 \% \mathrm{Cl}$ : $1.20-3.75$, followed by chronic pulmonary disease (adjHR: $1.81,95 \% \mathrm{Cl} 1.17-2.81$ ), chronic kidney disease (adjHR: 1.72, 95\% Cl: 1.13-2.62), diabetes mellitus (adjHR: $1.67,95 \% \mathrm{Cl}: 1.11-2.50)$, and atrial fibrillation (HR: 1.62; $95 \%$ Cl: $1.09-2.40$ (18). Besides several large international multicenter trials reported on percutaneous mitral repair of functional mitral regurgitation associated with HF $(19,20)$, registries from national or single centers provide valuable real-life results in unselected patients which may inform clinical decision making at a local level. Benak $A$ et al. (Cor et Vasa, Editor-in Chief: M. Aschermann) reported a cohort of 30 MitraClip implantations in patients with dilative cardiomyopathy and severe functional mitral regurgitation. The technical success was very high $(97 \%)$, with no 90 days mortality. During the 12 months clinical follow up, a significant improvement of functional class by NYHA, and quality of live (Kansas City Cardiomyopathy Questionnaire KCCQ), were reported. There was also reduction of left ventricular myocardial mass, and an increase systolic and diastolic arterial pressure. The mortality was $10 \%$ for the entire period of follow up (21). The technique 
broadens the treatment options for patients with severe functional mitral regurgitation who cannot undergo surgical repair because of valve disease characteristics and left ventricle dysfunction.

\section{Conclusion}

Most studies published in the high-ranking impact factor journals focus on drug therapy of HF and are devoted to a broad readership, but fail to characterize important local issues. Publications in the NSCJ, on the other hand, cover a wide spectrum of diagnostic and therapeutic modalities of HF, taking into account the national specificities of the problem. Most of these studies, however, are often single center and observational. That is the reason for the rather small number of included patients, and the lack of experimental models. However, information of HF strategies at a national level is eagerly required to help to implement the ESC clinical practice guidelines and optimize the care of patients with HF.

\section{References}

1. GBD 2017. Causes of death collaborators. Lancet 2018; 392: 1736-88.

2. Ponikowski P, Voors A, Anker SD, et al. 2016 ESC guidelines for the diagnosis and treatment of acute and chronic heart failure. Eur Heart J 2016; 37: 2129-2200. https://doi.org/10.1093/eurheartj/ehw128

3. Vaduganathan M, Claggett BL, Jhund PS, et al. Estimating lifetime benefits of comprehensive disease-modifying pharmacological therapies in patients with heart failure with reduced ejection fraction: a comparative analysis of three randomized controlled trials. Lancet 2020. https://doi.org/10.1016/S0140-6736(20)30748-0

4. Timmis A, Townsed N, Gale C, et al. European Society of Cardiology: Disease Statistics 2019. On behalf of the Atlas Writing Group. European Heart Journal 2020; 41: 12-85.

https://doi.org/10.1093/eurheartj/ehz859

5. Rossignol P, Hernandez AF, Solomon SD. Heart failure drug treatment. Lancet 2019; 393: 1034-44

https://doi.org/10.1016/S0140-6736(18)31808-7

6. Velazquez EJ, Morrow DA, DeVore AD, et al. Angiotensin-neprilysin inhibition in acute decompensated heart failure. N Engl J Med 2019; 380: 539-48. https://doi.org/10.1056/NEJMoa1812851

7. Wachter R, Senni M, BelohlavekJ, et al. Initiation of sacubitril/ valsartan in haemodynamically stabilised heart failure patients in hospital or early after discharge: primary results of the randomised
TRANSITION stydy. Eur J Heart Fail 2019; 21: 998-1007.

https://doi.org/10.1002/ejhf.1498

8. Wiviott SD, Raz I, Bonaca MP, et al. Dapagliflozin and cardiovascular outcomes in type 2 diabetes. N Engl J Med 2019; 380: 347-57. https://doi.org/10.1056/NEJMoa1812389

9. Fitchett D, Inzucchi SE, Wanner C, et al. Relationship between hypoglycaemia, cardiovascular outcomes, and empagliflozin treatment in the EMPA-REG OUTCOME trial. Eur Heart J 2020; 41: 209-17. https://doi.org/10.1093/eurheartj/ehz621

10. Badran HM, Elgharably MA, Faheem N. Clinical characteristics and in-hospital outcome of heart failure in women: a single center registry from Egyptian cardiac care unit. Egypt Heart J 2019; 71: 167. https://doi.org/10.1186/s43044-019-0032-0

11. Tomova V, Alexandrovna M, Atanasova M, et al. The polymorphism RS10455872 at the lipoprotein (a) gene locus enhances the risk of aortic valve disease. Bulgarian Cardiology Journal 2019; 13(4): 45-50.

12. Kaufmann CC, Hennenberg J, Bonderman D, et al. Transthyretin amyloidosis - update 2019. Austrian(?) J Kardiol 2019; 26(5-6): 112-6.

13. Vertes V, Nogradi A, Porpaczy A, et al. Left ventricular global longitudinal strain is impaired in systemic sclerosis and shows a significant correlation with the functional capacity of the patients. Cardiologia Hungarica 2019; 49:12-16.

14. Lelyavina TA, Sitnikova MY, Galenko VL, et al. The role of muscle tissue in the pathogenesis of chronic heart failure-the potential of exposure (FORMA study). Russ J Cardiol 2019; 58-65.

15. Vdovenko DV, Libis RA. Assessment of the functional state of left heart in patients with chronic heart failure with preserved ejection fraction. Russ J Cardiol 2019; 2: 26-30. (In Russ)

16. Içen YK, Demirtas AO, Koç AS, et al. Liver stiffness value obtained with ElastPQ ultrasound increases with NYHA class in chronic heart failure patients and reduced ejection fraction. Turk Kardiyol Dern Ars 2019; 47(4): 281-3. doi: 10.5543/tkda.2018.62282

17. Öztürk S, Gürbuz AS, Kirma C. Relationship between SYNTAX score and myocardial viability in ischemic cardiomyopathy. Turk Kardiyol Dern Ars 2019; 47(5): 350-6. https://doi.org/10.5543/tkda.2019.88054

18. Guedeney P, Huchet F, Manigold T, et al. Incidence of, risk factors for and impact of readmission for heart failure after successful transcatheter aortic valve implantation. Arch Cardiovasc Dis 2019; 112; 765-72. https://doi.org/10.1016/j.acvd.2019.09.008

19. Aila Wadi G, Lim DS, Mack MJ, et al. One-year outcomes after MitraClip for functional mitral regurgitation. Circulation 2019; 139: 37-47. https://doi.org/10.1161/CIRCULATIONAHA.117.031733

20. Pibarot $P$, Delgado V, Bax JJ. Mitra FR versus COAPT: lessons from two trials with diametrically opposed results. Eur Heart J Cardiovasc Imaging 2019; 20: 620-624. https://doi.org/10.1093/ehjci/jez073 21. Benak A, Skalicka B, Haskova J, et al. MitraClip in patients with functional mitral regurgitation and advanced heart failure - Single centre experience. Cor et Vasa 2019; 61: 8-14. 